CAHIERS DE

NARRATOLOGIE

\section{Cahiers de Narratologie}

Analyse et théorie narratives

25 | 2013

Humour et modernité dans les littératures de langues romanes du XIXe au XXIe siècle

\title{
Aux origines de la notion d'humour en Italie au XIXe siècle
}

\section{Edwige Comoy Fusaro}

\section{(2) OpenEdition}

\section{Journals}

Édition électronique

URL : http://journals.openedition.org/narratologie/6784

DOI : 10.4000/narratologie.6784

ISSN : 1765-307X

Éditeur

LIRCES

Référence électronique

Edwige Comoy Fusaro, " Aux origines de la notion d'humour en Italie au XIXe siècle », Cahiers de Narratologie [En ligne], 25 | 2013, mis en ligne le 20 décembre 2013, consulté le 19 avril 2019. URL http://journals.openedition.org/narratologie/6784; DOI : 10.4000/narratologie.6784 


\title{
Aux origines de la notion d'humour en Italie au XIXe siècle
}

\author{
Edwige Comoy Fusaro
}

1 Cette communication a pour objectif de tenter de cerner ce que recouvre le terme d'humour à la moitié du XIXe siècle en Italie, lorsqu'il apparait sous la plume des écrivains, journalistes et essayistes. Il s'agit d'une recherche in fieri, qui prend naturellement appui sur les études préalables sur l'écriture humoristique italienne au XIXe siècle, notamment les travaux réunis par Giancarlo Mazzacurati sur la littérature humoristique ${ }^{1}$, la monographie de Roberta Colombi sur l'humour dans la littérature et les publications périodiques ${ }^{2}$, l'enquête d'Anna Scannapieco sur les différentes acceptions du lexème dans l'usage ${ }^{3}$. À ce jour, néanmoins, la plupart des recherches ont porté sur une tradition humoristique littéraire établie a posteriori, c'est-à-dire en qualifiant d'humoristique un corpus d'œuvres ou de textes qui n'étaient pas désignés comme tels par leurs auteurs. Il est indéniable qu'une écriture humoristique italienne apparaît bien avant que le terme ne fasse son entrée dans les livres et revues. Plusieurs généalogies ont été esquissées qui établissent les auteurs que l'on peut considérer comme des humoristes avant la lettre : Leopardi ou Manzoni, par exemple ${ }^{4}$. Cependant, aucune étude de fond n'a été menée sur le sens du terme dans les écrits explicitement qualifiés d'humoristiques. À ma connaissance, seul l'article d'Anna Scannapieco - paru il y a un peu plus de dix ans propose une premier défrichement de cette question et je me propose ici d'apporter une pierre à l'édifice qu'elle a commencé à bâtir.

Dans le sillage de son enquête, on peut établir un certain nombre de repères chronologiques sur la base des premières occurrences repérables dans les dictionnaires, les publications périodiques et les œuvres littéraires du terme umorismo [humour] et de ses dérivés umoristico [humoristique] et umorista [humoriste] dans un autre sens que le sens médical issu de la théorie des humeurs de Galien, qui était l'acception en vigueur auparavant $t^{5}$. Dès les premières - rares - occurrences, sous la Restauration, l'humour s'impose d'emblée comme une qualité du texte littéraire en lien avec une caractéristique de l'auteur: la versatilité de ce dernier se reflèterait dans une œuvre hétérogène et 
hétéroclite. Ainsi, en 1827, Paride Zajotti utilise-t-il le terme umoristico au sujet des romans bâtis sur le modèle des œuvres de Richter. L'année 1848 marque un tournant. À partir de cette date, l'humour est étroitement associé à un genre journalistique, celui de la presse satirique, qui prend son essor dans les grandes villes du nord de la péninsule: Milan, bien sûr, mais aussi Florence, Trieste ou Venise. En 1848-1849 paraissent les premiers périodiques dont le sous-titre comporte le terme umoristico ${ }^{6}$. Voient ainsi le jour Lo Spirito folletto. Giornale diabolico, politico, umoristico, comico, critico, pittoresco à Milan, Il diavoletto : giornale diabolico, politico, umoristico, comico e se occorresse pittorico à Trieste, la Lanterna magica. Giornale diabolico, umoristico, pittoresco et Lo specchio: giornale politicoumoristico-morale à Florence, ou encore La frusta : giornale né diabolico, ma critico, umoristico, teatrale, comico e un pochino poetico e bernesco à Trieste ${ }^{7}$, et bien d'autres. Jusqu'alors, le lexème n'est répertorié que sous forme adjectivale (umoristico $\left.{ }^{8}\right)$. C'est en revanche dans un livre qu'apparait la première occurrence du terme umorismo: dans les Confessioni di un italiano d'Ippolito Nievo [1857-1858, posthume]. Au même moment, on trouve l'adjectif umoristico dans un autre roman: le Viaggio di un ignorante, ossia ricetta per gli ipocondriaci [1857] de Giovanni Rajberti. Le terme ne qualifie plus alors seulement un genre littéraire ou journalistique mais un style, un ton, un état d'esprit. Nous y reviendrons dans un deuxième temps.

Les premiers périodiques qui se déclarent explicitement humoristiques ayant déjà fait l'objet d'études, mes recherches ont porté sur les hebdomadaires des années 1850, en particulier : La lente. Giornale umoristico. Lettere, scienze, arti, commercio, industria, teatri, le Pasquino. Giornale umoristico, non politico, con caricature et L'uomo di pietra. Giornale letterario, umoristico-critico, con caricature. Le premier, La lente, paraît en 1856 à Florence comme hebdomadaire : il se transforme en quotidien en 1861, sous le titre Gazzetta del popolo. Le Pasquino, quant à lui, voit le jour en 1856 à Turin. Il prend son nom de l'une des « statues parlantes » de Rome, statues sur lesquelles les Romains avaient coutume d'afficher des messages satiriques anonymes contre des personnages publics du gouvernement ou de la Curie (les pasquinate). L'uomo di pietra, enfin, apparaît en 1856 à Milan. Il revendique sa descendance du Pasquino, le titre faisant explicitement écho à une statue qui se trouve sous les arcades du corso Vittorio Emanuele à Milan, comparable à la statue romaine du Pasquino. Les Milanais avaient également coutume d'y afficher des mots satiriques'. À ce corpus nous ajouterons la Cronaca grigia de Cletto Arrighi (hebdomadaire fondé en 1860 à Milan), où l'on trouve de précieuses réflexions sur l'humour - quoique le journal se défende d'être humoristique -, et deux pages de romans, celles où - sauf erreur de ma part- le terme d'humour apparaît pour la première fois en littérature: dans les Confessioni di un Italiano de Nievo et le Viaggio di un ignorante de Rajberti.

4 Le dépouillement des périodiques autoproclamés humoristiques permet de poser les jalons du genre, un genre qui est aussi un style d'écriture. C'est ce que nous verrons dans un premier temps, avant de nous pencher sur les publications non revendiquées comme telles, mais qui fournissent de premières définitions de l'humour d'autant plus intéressantes qu'elles sont polémiques. Ainsi pourra-t-on, au terme de cette brève enquête, élucider l'ambivalence de ces lignes de la main Cletto Arrighi dans L'uomo di pietra:

les Anciens connaissaient tout, sauf peut-être le cosmétique hongrois pour fixer les moustaches, et cet absurde style d'écriture qu'on appelle humoristique, dont on me dit qu'il plaît beaucoup au public cultivé, et que j'appellerais personnellement sot et grotesque si ne j'avais pas de bonnes raisons de dissimuler mes sentiments en la matière ${ }^{10}$. 
Si les acceptions non médicales de l'humour ont fait une apparition si tardive en Italie, alors qu'une écriture humoristique existait déjà bel et bien, c'est probablement en raison de son caractère d'importation. Le glissement sémantique s'était imposé dans la langue anglaise aux XVIIe-XVIIIe siècles ${ }^{11}$, puis dans la langue française à la fin du XVIIIe siècle ${ }^{12}$. En italien, au contraire, il faut attendre la moitié du XIXe siècle. Les milieux culturels de la péninsule souffraient alors du rayonnement des nations voisines - même à Milan, capitale du Royaume lombard-vénitien en pleine expansion ${ }^{13}-$, comme en témoigne la polémique que déclencha le célèbre article de Madame de Staël « Sulla maniera e l'utilità delle traduzioni », c'est-à-dire sur l'opportunité de traduire les œuvres étrangères, paru dans le premier numéro de la Biblioteca Italiana en janvier 1816. L'usage tardif du terme d'humour dans un sens non humoral est vraisemblablement dû à la réticence des Italiens vis-à-vis d'un mot perçu comme étant barbare, au sens premier, c'est-à-dire étranger, voire même au sens figuré : étrange, macaronique. Nous verrons bientôt que l'apparition du terme en littérature se fait précisément sous le signe de la comparaison avec les autres cultures européennes.

7 La Lente, le Pasquino et L'uomo di pietra sont clairement inspirés des journaux français et anglais Le Charivari et Punch (fondés respectivement en 1832 et 1841, le second - un hebdomadaire, à la différence du quotidien satirique parisien - étant la version anglaise du premier : son titre original était The London Charivari). Il n'est pas anodin que le terme d'humour apparaisse tout d'abord dans les publications périodiques. Le marché éditorial, dont la croissance ne cesse de s'affirmer parallèlement à une forte diversification, est alors en train d'asseoir ses bases proto-capitalistes ${ }^{14}$. Au regard des publications livresques, le journal a un statut marginal, puisqu'il est destiné à un usage immédiat. Par nature, c'est un produit jetable. Il n'est guère surprenant qu'un genre aussi douteux que le genre humoristique - dépourvu de racines autochtones - n'ait pu, in primis, venir à bout des réticences des gens de lettres que dans le cadre d'un système de communication éphémère et familier. Ainsi explique-t-on l'ancrage municipal et la prédilection pour les sujets d'actualité principalement anecdotique de la vie urbaine contemporaine.

Genre douteux, le genre humoristique l'est aussi parce qu'il est bas, impur (c'est-à-dire mixte) et voué au rire. Ces caractéristiques aussi sont issues des modèles d'importation. Dans un numéro de 1711 du célèbre The Spectator, le premier journal d'opinion européen, on trouve une définition généalogique de l'humour ${ }^{15}$. Addison pointe le mélange de sérieux et de léger dans l'humour, celui-ci étant le fruit du mariage de l'esprit [Wit] et de la gaieté [Mirth]. Néanmoins le rire l'emporte toujours, écrit-il, quoique le plus lointain aïeul de l'humour soit la vérité [Truth], mère du bon sens [Good Sense] : « he never fails to make his Company laugh » [Il ne manque jamais de faire rire la compagnie]. Légèreté et désinvolture caractérisent également les premières formes d'humour auto-proclamé en Italie telles qu'elles apparaissent dans les hebdomadaires des années 1856-1858. C'est pourquoi tous ces journaux ont en commun d'être illustrés, souvent par des dessins caricaturaux ${ }^{16}$ - comme auparavant le Charivari - ; c'est aussi pourquoi la plupart des auteurs ne signent que par des sobriquets: BTZ, PIF, FSCHT ou BRRRR..., par exemple, dans le Pasquino. Ainsi trouve-t-on dans la rubrique «Braciere» [Brasero] de L'uomo di pietra des histoires drôles comme celle-ci :

- Tu es sorti de bien bonne heure ce matin, car j'ai trouvé ta porte close à neuf heures.

- As-tu tiré la sonnette? 
- Parbleu ! Je l'ai secouée pendant une demi-heure.

- Voilà le tort. Je t'ai pris pour un créancier ${ }^{17}$. publications, qui est affichée dans les sous-titres eux-mêmes, est leur nature hétérogène. Certains périodiques utilisent manifestement l'accumulation de qualificatifs variés comme un ressort comique autonome, comme L'Inferno [L'Enfer] de Livourne, dont le sous-titre est : giornale della notte: politico-diabolico-bizzarro-letterario-umoristico-fantasticoridicolo-critico-satirico-pittoresco-teatrale : illustrato da mille e più disegni all'anno [journal de la nuit: politique, diabolique, bizarre, littéraire, humoristique, fantastique, ridicule, critique, satirique, pittoresque, théâtral : illustré de plus de mille dessins par an]. Cet aspect est dû au lien qui unit étymologiquement l'humour à l'humeur ${ }^{19}$, mais aussi à la nature même du genre. Car l'humour est censé refléter l'ensemble multicolore de la vie, d'une part, et, d'autre part, dévoiler la face cachée des choses et des hommes ${ }^{20}$. Déjà Sterne, en son temps, affirmait que le page en marble-paper insérée dans son roman était l'emblème motly de son œuvre : bariolé et bouffonesque ${ }^{21}$.

\section{L'humour journalistique : un genre satirique}

11 Toutefois, sous son apparence débonnaire et superficielle, l'humour comme genre journalistique est génétiquement polémique, conformément à l'esprit du Charivari, du Punch et des pasquinate qui avaient inspiré ces hebdomadaires. Les périodiques des années 1848-1849 affichaient d'emblée des visées essentiellement satiriques, ne serait-ce que dans leurs titres, au travers des figures de diablotins (Il diavoletto [le diablotin] de Trieste) ou d'autres métaphores explicites (La frusta [le fouet] de Trieste, La zanzara [le moustique] de Florence). Il en va de même dans les dernières années de la période pré-unitaire, même si les tons sont plus nuancés, à cause du contexte politique, comme l'explique Ugo M. Olivieri :

Les années révolutionnaires 1848-1849 se présentent comme une contingence historique particulière durant laquelle la liberté de la presse retrouvée et la présence d'options politiques différentes sur la question nationale déterminèrent une orientation résolument politique et militante des très nombreux titres qui naquirent dans tous les États italiens

tandis que la fin de l'expérience révolutionnaire et le retour de la censure obligea les périodiques à reprendre le «modèle du journal littéraire et de mœurs ${ }^{22}$ ». En effet, la satire que la Lente, le Pasquino et L'uomo di pietra déploient est essentiellement une satire de mœurs. Les cibles privilégiées sont les modes en vigueur dans la bonne société et les travers de certains personnages publics ou de certains groupes sociaux bien identifiés. Le programme de La lente ne laisse aucun doute :

Cahiers de Narratologie, 25 | 2013 
Se présenter avec une Loupe au beau milieu du Siècle des Lumières, où l'on est en droit de présumer que tous les hommes voient clair, et qu'il n'est plus possible de prendre des vessies pour des lanternes, comme cela arrivait souvent à nos défunts Ancêtres, pourrait éventuellement être jugé comme une entreprise inutile ou railleuse ${ }^{23}$.

Jouant sur la multiplication des lunettes et binocles en tout genre dans les lieux les plus en vue de Florence, l'éditorialiste conclut qu'un "Journal-Loupe apportera un grand bénéfice à l'humanité aveugle ${ }^{24} »$. Le but du journal est de « chercher la vérité par la voie du ridicule ", de "découvir des nouveautés et les publier en riant ${ }^{25}$ ", de «sonder les plaies de la sociétée ${ }^{26} »$, une société qui, en dépit des discours sur les progrès de l'humanité civilisée et le triomphe des lumières de la raison, demeure malade, rivée comme elle l'est aux plaisirs matériels ${ }^{27}$. Ce sont donc surtout de brefs textes sarcastiques qui remplissent les colonnes des journaux humoristiques. Ils ont généralement un caractère narratif et font grand usage de l'ironie. Dans la rubrique des "scènes de carême " de La lente, par exemple, le journaliste met en scène un jeune étudiant en médecine qui réclame de l'argent à son père, arguant de la cherté des livres et des instruments chirurgicaux, feignant une application sans faille dans ses études alors qu'il ne songe qu'à s'adonner à des amusements coûteux et licencieux :

Lisons. «Très cher Père. Je jouis enfin d'un peu de calme. Le fracas du carnaval, le bruit des voitures, le tapage des gens masqués me laissent à peine les heures de la nuit pour me consacrer à mes études. »

Notez qu'il a hurlé sous son masque tous les jours et plus encore toutes les nuits. De plus, il a dansé toutes les nuits jusqu'au petit matin.

[...]

«Les instruments que j'ai achetés grâce à l'argent que vous m'avez envoyé la dernière fois... »

Il a acheté des éperons pour monter à cheval.

«Sont excellents, mais je devrai encore dépenser de l'argent pour les faire aiguiser, car en quelques jours j'ai coupé une douzaine de jambes et de cuisses... »

Des jambes et des cuisses de poulet ${ }^{28}$.

14 Autre exemple, le portrait du type contemporain de l'inventeur dans la rubrique des « nouveaux types sociaux » du Pasquino:

Il y a deux sortes d'inventeurs. L'inventeur qui naît inventeur, comme l'on naît poète ou artiste, et l'inventeur qui devient inventeur, à la suite des expériences de la vie, après avoir essayé... un peu de tout.

Le premier est souvent un martyr, quand ce n'est pas un fou. C'est un original, qui vit d'idées fixes, de pain et d'eau. Ou il invente quelque chose de grand, comme Gutenberg [...], ou bien il meurt à l'hôpital sans avoir rien inventé ; c'est-à-dire - notez bien - qu'il meurt à l'hôpital dans les deux cas [...].

Ce type-là n'est pas l'Inventeur que je préfère, ni celui que l'on rencontre le plus fréquemment de nos jours. [...] Aujourd'hui la première chose à laquelle s'applique sérieusement un inventeur - qu'il soit poussé par le génie ou par la charlatanerie -, c'est faire en sorte de ne pas mourir à l'hôpital. Et même, que dis-je ?... Les choses se sont tant et si bien inversées que, presque toujours, l'Inventeur, à plus forte raison le charlatan sans génie, non seulement ne meurt pas à l'hôpital, mais laisse cent mille lires à ses héritiers pour fonder un hôpital..., lequel hôpital - par parenthèse - est construit avec les briques des actionnaires qui, à cause de l'Inventeur, meurent souvent à l'hôpital à sa place ${ }^{29}$.

15 Au fil des historiettes, boutades et sotties, ce sont tous les vices les plus courants de la faune urbaine qui sont croqués, présentant une galerie de vaniteux, d'hypocrites, d'imposteurs, de jouisseurs de tout poil, hommes et femmes confondus. Les attaques personnelles sont cependant rares, contrairement aux attaques typologiques derrière 
lesquelles se livre en réalité une critique acerbe de la société des plaisirs et des discours lénifiants, des apparences honnêtes qui dissimulent les motivations véritables inavouables. Satire de mœurs, donc, mais aussi satire culturelle.

La dérision frappe également, pour une part substantielle, les œuvres littéraires et leurs auteurs. Le tour narratif des textes est déjà un indice du goût des journalistes pour les lettres. De fait, les rubriques consacrées aux sujets littéraires et les références culturelles qui émaillent les proses confirment que ces journaux, malgré la superficialité affichée, émanent de personnages issus des milieux cultivés (le contraire serait étonnant) et, surtout, s'adressent à un public averti. Un article de La lente, signé du prête-nom Mastro Forese [Maître Paysan], dos à dos Romantiques et Classiques pour aboutir à un éloge d'un genre trop peu fréquenté, à son goût : le genre satirique, genre « du plus haut intérêt, qui ne vieillit jamais » et dont l'objet doit être de brosser « les mauvaises mœurs »; et de citer Giuseppe Giusti en guise de modèle insurmontable du genre ${ }^{30}$. Un article plus polémique du Pasquino critique quant à lui les romans d'Alexandre Dumas :

Le caoutchouc! - substance merveilleuse, surprenante, philanthropique ! [...]

Mais de tous les miracles que l'homme a fait faire jusqu'à présent à ce produit végétal, le plus grand, le plus utile, est - sans aucun doute - son application à la littérature et à l'art éditorial.

Et ce miracle aussi, le monde civil le doit à la France, à la généreuse France, à la grande nation civilisatrice. [...]

La dynastie des Alexandres Dumas fut la première à appliquer le caoutchouc au roman.

Alexandre I vous pond un roman de trois cents pages en vingt-quatre heures. [...] Ouvrez n'importe lequel de ses romans parmi les cent mille qu'il a écrits et vous pourrez vous en convaincre.

Exemple :

Marceline!

Albert !

Ah!

Oh!

C'est bien toi?

Oui.

Toi?

Bien moi.

Grand Dieu!

Pourquoi es-tu surpris?

Je te croyais morte.

Morte?

Morte !

Ah, Albert !

Oh, Marceline!

[...]

17 À la page dix-sept Albert se convainc finalement que Marceline n'est pas morte et c'est la fin du chapitre.

Puissance du caoutchouc ${ }^{31}$ !

Dans un esprit plus polémique encore, Cletto Arrighi s'adonne à une critique acerbe de l'ignorance des professeurs à l'occasion du soi-disant compte rendu d'un leçon entendue à la Faculté des Lettres de Paris sur Dante :

Dante doit sa renommée - il suo grando nome - non pas à son style - non è lo stile che gli ha fatto honore - ni à sa Divina Comedia - ni à son exil du Royaume de Naples - ni à sa femme Béatrix - mais il la doit... chose étrange! il la doit à son 
commerce de peaux de lapin [...].

Il avait ouvert un magasin de cuirs de Russie et de peaux de lapin, qui de son nom furent appellées en Italien pelli di Dante, espèce de peau dont on fait les pantalons pour les postillons et les gants pour la gendarmerie. [...]

La Société Alighieri et $\mathrm{C}$. prospera à l'aide du journal qu'était un puissant moyen de publicité. La preuve évidente de cette vérité résulte clairement, quand on pense qu'on y trouve souvent la description des articles de son commerce. Dans le premier chant, par exemple, on trouve ces vers :

Ed ecco quasi al cominciar di l'erta [Mais voici, presque au début de la montée,]

Una lonza leggera e presta molto [une panthère légère et très agile,]

Che di pel maculato era coperta. [que recouvrait un pelage moucheté]

Vous voyez la subtilité de cette annonce; c'était une manière un peu détournée d'avertir le public qu'il tenait un assortiment de pelli di Dante maculées pour les manchôns - manière dètournée qu'il employait pour ne pas payer les droits de Finance, qui étaient alors bien lourds et insupportables. [...]

Un autre argument à prouver que Dante n'était pas le poète célèbre, que l'enthousiasme et la folie de ses compatriotes l'on crée en suite de temps, parce qu'ils n'entendaient rien au sens caché de son journal, c'est que le Comte Ugolino, qu'était contemporain de Dante, et qu'aurait dû le connaître, ne le connaissait pas du tout :

Io non so chi tu sie.

Lui dit le Comte, levant la bouche de l'assiette, pendant qu'il dînait avec l'archevêque Ruggeri ${ }^{32}$.

Néanmoins, derrière le ton facétieux et la légèreté, la polémique est de nature politique.

Le thème est annoncé dans les sous-titres journaux, comme Lo specchio de Florence: giornale politico-umoristico-morale [journal politique, humoristique, moral], ou Il gobbo di Rialto : Giornale critico politico umoristico urbano [journal critique, politique, humoristique, municipal]. Dans La lente, Pasquino et L'uomo di pietra, la politique est a priori exclue ( Pasquino précise même: Giornale umoristico, non politico, con caricature), mais les allusions aux affaires publiques ${ }^{33}$ n'en sont pas moins légion. L'expérience récente de la guerre de Crimée hantait sans doute encore les esprits lorsque le Pasquino publia la nouvelle Un amore in Crimea [Un amour en Crimée], où l'on narre la mésaventure burlesque d'un soldat qui, s'étant entiché d'une vivandière en raison de l'abstinence à laquelle il était contraint, et non en raison des attraits - plus que grossiers - de celle-ci, lui avait offert à manger et à boire sans parvenir à ses fins. La guerre sert de simple décor. En effet, les événements politiques sont souvent relégués à l'arrière-plan et traités de manière prétextuelle. À ce titre, le programme du Pasquino est éclairant. Il se présente sous une forme narrative. Protagoniste :

Lippa, Ministre plénipotentiaire de Sa Majesté le Roi de Cappadoce, en mission spéciale auprès du Senatus Populusque Romanus, auquel il devait communiquer une note énergique de son Gouvernement au sujet d'une espèce de question d'Orient de l'époque ${ }^{34}$.

20 Dans la rue, Lippa suscite les rires des passants. L'une des personnes qui se gaussent finit par lui en révéler la raison: son visage est souillé d'encre. Lippa se nettoie aussitôt et remercie chaleureusement son interlocuteur : sans lui, la guerre était inévitable, dit-il. En repensant à son aventure,

Lippa comprit l'utilité d'un éclat de rire opportun ; et songeant alors à l'efficacité et à l'importance de la mission philosophico-humanitaire du rire, il en arriva à l'idée de publier une Ephéméride comique avec caricatures. [...]

21 Ainsi vit le jour le premier journal humoristique dont l'histoire se souvienne ${ }^{35}$. 
confia la direction du journal à Pasquino, l'homme qui lui avait expliqué le motif de la bonne humeur qu'il avait suscitée ; l'un des hommes, notons bien, qui « étaient en train de discuter de politique à l'entrée d'une boutique de café », précise opportunément le narrateur.

23

politique est effectivement au cœur des préoccupations des journaux humoristiques. D'ailleurs, l'humour est régulièrement présenté comme une arme. Il est ainsi question d'une "épée à double tranchant ${ }^{36}$ " dans La lente. Dans le même esprit, L'uomo di pietra propose une définition belliqueuse de l'humour :

L'humour est une bataille livrée avec des mots, avec de fines boutades...

[...].

L'humour est une protestation forte et généreuse contre les hommes, les choses, les œuvres mauvaises; c'est un reproche adressé aux préjugés nocifs; c'est un redressement spirituel des idées fausses; c'est un désir de mieux dans tous les rapports humains, exprimé avec force par l'apologue, le symbole, l'image, le hiéroglyphe, le sous-entendu ; c'est une condamnation de l'obscur idéal contre la turpitude acclamée, imitée ; c'est un coup ferme asséné au fatras de tout ce qui est rance, absurde, vil ; c'est une guerre de mots et de choses, d'idées et de faits ${ }^{37} \ldots$

Or, les métaphores belliqueuses ne sont pas simplement ornementales. Les hebdomadaires des années 1850 abordent assez timidement les questions politiques, mais à mesure que les événements qui devaient marquer le dernier chapitre du processus risorgimentale s'accélèrent, les auteurs traitent de ces questions avec plus de continuité et d'ardeur. Une fois l'épopée garibaldienne accomplie, Cletto Arrighi est très clair :

Cette stratégie qui, comme la stratégie militaire, a ses offensives et ses replis, ses embuscades et ses stratagèmes, ses surprises, ses fausses attaques, a été appelée par un nom presque anglais, humour, qui est d'autant plus vif et léger dans la forme qu'il est plus sérieux dans le fond; un instrument qu'on devra jeter lorsque la phrase pourra être librement explicite, courageuse, entière, au lieu de celle qu'une sotte censure ne nous permet presque même pas de penser aujourd'hui. On croit généralement que l'humour est ce je ne sais quoi, en littérature, par lequel on fait rire les lecteurs. Oui ; le rire aussi est une belle chose, lorsqu'il le faut, et heureux les écrivains qui savent le susciter lorsqu'il le faut. Cependant il ne s'agit là que d'une manière spéciale d'humour, tout au plus; mais qui ne comprend pas tout le concept dans son entier. [...]

25 La menace continuelle d'être frappés de censure que subissent les journaux militants, la fièvre des lecteurs à dénicher la signification cachée des phrases qui sont déjà passées sous les yeux des stupides commissaires de censure, et tout ce travail d'interprétation, enfin, qui fait pour ainsi dire du lecteur un collaborateur et un complice des journalistes... tout cela contribue à vivifier et à maintenir éveillé l'esprit littéraire dans ma ville asservie ${ }^{38}$.

Ce passage est tiré d'une livraison de Cinque mesi. Memorie d'un ex repubblicano, roman que Cletto Arrighi affirme avoir simplement adapté du manuscrit d'un déserteur qu'il avait hébergé un soir avant qu'il ne parte rejoindre les chemises rouges. En note, le journaliste précise :

Je ne suis pas totalement de l'avis de mon ex-républicain. L'humour peut aussi servir dans la presse libre, dans la mesure où il peut ouvrir la voie à certaines idées que les journaux sérieux et prudents n'auraient pas le courage d'exprimer, et encore moins de défendre. Je veux bien parier que la Perseveranza n'oserait pas parler aussi librement aujourd'hui des choses les plus sacrées dans le vieux système si depuis déjà longtemps le Fischietto, l'Uomo di Pietra et le Pungolo (humoristique) ne lui avaient pas ouvert la voie en détruisant par le ridicule le prestige dont certains 
noms jouissaient encore auprès de la majorité de la population. Par leur humour, les journaux de caricature préparent pour ainsi dire les esprits à un langage officiel, plus passionné et plus efficace, ce pour quoi on pourrait dire d'eux que ce sont les avant-coureurs de l'opinion publique ${ }^{39}$.

Les journaux humoristiques entendaient donc servir les intérêts patriotiques du Pays. Dans les régions où sévissait encore une domination étrangère, comme c'était le cas du Royaume lombard-vénitien avant la deuxième guerre d'indépendance de mai 1859, l'humour apparaissait comme un instrument de contournement de la censure. Les critiques sont unanimes pour établir un lien très serré entre les crises politiques et l'émergence de l'humour. Anna Scannapieco déduit des données chronologiques que la qualification d'humour implique une conscience de la crise $\mathrm{s}^{40}$. Daniela Marcheschi relie étroitement la naissance d'un grand nombre de journaux satiriques français à la révolution de 1830. Elle observe par ailleurs que 1848 marque un tournant dans l'écriture humoristique en cela que cette écriture se déporte de la littérature d'ascendance sternienne pour investir le journalisme satirique ${ }^{41}$. Genre de seconde catégorie vis-à-vis des journaux sérieux, faisant tout au mieux office de rabatteur, relégué dans les marges de l'activité éditoriale, du point de vue stylistique le journalisme humoristique montre cependant une certaine vivacité, annonciatrice, elle aussi, d'une écriture qui devait s'affirmer quelques années plus tard au sein de la Scapigliatura.

\section{L'humour journalistique : un style d'écriture}

On se souvient du passage des "scènes de carême ", dans La lente, qui fait le jour sur les mensonges d'un jeune étudiant en médecine à son père :

Lisons. «Très cher Père. Je jouis enfin d'un peu de calme. Le fracas du carnaval, le bruit des voitures, le tapage des gens masqués me laissent à peine les heures de la nuit pour me consacrer à mes études. »

Notez qu'il a hurlé sous son masque tous les jours et plus encore toutes les nuits. De plus, il a dansé toutes les nuits jusqu'au petit matin ${ }^{42}$.

Et caetera. Le journaliste intercale à la retranscription de la lettre du jeune homme à son père des commentaires personnels démystifiants. Le procédé est courant dans le journal florentin. On le retrouve dans un autre numéro, où il est question d'une famille à la mode :

Hier soir Madame la mère est allée au théâtre, où l'on a représenté un drame français. Une fois rentrée chez elle, dans le silence de la nuit, dans l'obscurité de la chambre, les scènes du drame repassent devant les yeux de son esprit. (J'ouvre une parenthèse. Puis-je en bonne conscience donner des yeux à l'esprit ?... Pourquoi pas !... Pétrarque, que Dieu lui pardonne les Pétrarquistes, lui a bien donné des genoux... Rasséréné, je ferme la parenthèse ${ }^{43}$ ).

L'article est significativement signé Yorick, en référence évidente à l'alter ego de l'auteur du Sentimental Journey. De fait, Sterne est coutumier de tous les artifices que les journalistes humoristes italiens emploient: la digression, l'intrusion du je, le commentaire méta-narratif, le ton désinvolte et familier, mais aussi l'interpellation du lecteur, autre procédé utilisé par l'anonyme auteur du portrait mondain :

Et le mari ?... direz-vous, lecteurs très intelligents ? Le mari a fait un petit voyage en

France, et il est revenu habillé à la dernière mode ${ }^{44}$.

30 La mise à nu des artifices de l'écriture et des conventions romanesques est au rang des procédés régulièrement rencontrés dans les colonnes des périodiques. Ainsi, le programme du Pasquino s'ouvre sur ces lignes: 
Un beau matin de... (l'année n'est pas sûre, ce qui est sûr c'est que c'était un beau matin de mai, mois où tous les matins sont beaux - quand il ne pleut pas) ${ }^{45}$. du Pasquino sur le caoutchouc appliqué au roman français. Mais il serait fastidieux et superflu de passer en revue l'ensemble des effets stylistiques directement inspirés du roman de Sterne et, plus généralement, de la tradition littéraire anglo-saxonne (songeons notamment à Richter) que les journalistes des hebdomadaires humoristiques italiens reprennent à leur compte. Il suffit sans doute de relever que, là aussi, ces périodiques jouent le rôle d'éclaireurs d'un terrain qui sera ensuite investi par la littérature. On observe en somme dans ces formes expérimentales le paradoxe soulevé par Franco Brioschi et Costanzo Di Girolamo au sujet de la naissance du roman (dont le plus éminent ailleul est, selon eux, le Don Quichotte) : une naissance due à des motivations antilittéraires, dans le but de dénoncer les mensonges d'une tradition désormais détachée de l'expérience réelle et de restituer leur vérité aux choses, mais qui marque également le début d'une tradition méta-littéraire, où l'auteur s'attache à dévoiler ostensiblement les mécanismes et les artifices du récit, pour mieux en dénoncer le caractère conventionnel ; de sorte que «le moment anti-littéraire se transforme en son contraire et complémentaire : le moment méta-littéraire ${ }^{46} »$.

\section{Le vilain petit canard de la modernité 47}

Dans les mêmes années, en littérature, justement, l'humour explicitement désigné apparaît dans Il viaggio di un ignorante ossia ricetta per gli ipocondriaci et dans les Confessioni di un Italiano. Nievo qualifie d'humoristiques les conversations du sénateur Almorò Frumier et de ses amis de Portogruaro. Il distingue l'humour méridional de l'humour septentrional :

Ceux qui ont cherché les fondateurs de l'humour en Angleterre n'ont certainement jamais vécu à Venise et ne sont jamais passés à Portogruaro. Ils y auraient trouvé cet humour méridional qui est le fruit d'une longue oisiveté séculaire, d'excellents estomacs et d'esprits vifs, joyeux, éveillés; cet humour qui se distingue autant de l'humour nordique que le brouillard nocturne des marécages se distinguent de l'horizon lumineux et vaporeux d'un beau coucher de soleil d'été. Comme le nordique, il méprise la vie et les choses qui sont en elle; c'est là leur lien de parenté ; mais, pour cette raison même, il les traite de façon insouciante et joyeuse ; c'est là leur différence ${ }^{48}$.

Limité à ces quelques lignes, le jugement du narrateur pourrait sembler élogieux: l'humour qui fleurit sur la terre ensoleillée est plein de vie et de gaieté. Néanmoins, dans les pages précédentes, la description des usages du milieu aristocratique provincial où il prend racine ne laisse aucun doute sur la nature critique du regard qu'y porte l'auteur. Le noble Frumier fait partie de ceux qui, à l'automne 1788, quittent Venise, où l'on redoute une répercussion des événements de Paris (la convocation des États généraux y avait été décrétée en juillet). Comme dans les hebdomadaires satiriques, le contexte politique est grave et cette même gravité semble susciter le repli sur l'humour. Dès la description de l'arrivée de la noble famille à Portogruaro, le narrateur adopte un ton ironique :

Le noble patricien prit congé avec l'affabilité proverbiale des Vénitiens. De l'évêque au jardinier, personne ne fut privé de la faveur de son sourire ; il baisa l'anneau du premier, et c'est avec la même modestie qu'il donna au second une calotte ${ }^{49}$. 
L'hypocrisie règne dans la petite société qui anime la maison de villégiature du sénateur et, lorsque les invités sont partis, on y dit « beaucoup de bien de soi, un petit peu de mal de ceux qui étaient partis » mais "la discrétion vénitienne ", précise le narrateur, «se contente de tailler des costumes sans ronger les chairs jusqu'à l'o ${ }^{50}$ ». La médisance reste donc modérée. Pareillement, les considérations du narrateur sur l'humour méridional soulignent sa disposition fondamentale à l'allégresse. Nievo insiste sur la frivolité et l'hétérogénéité des conversations :

la petite académie du Sénateur se regroupait dans un angle du salon pour bavarder de politique, et pour commenter les nouvelles les plus scandaleuses de la ville. C'était une musique des plus variées, un véritable opéra semiseria, plein de motifs ridicules et sublimes, bouffes et sérieux [...] ; et aux raisonnements graves on ne cessait d'alterner la plaisanterie et le rire. Tout prétexte à amusement était bon ${ }^{51}$.

En d'autres termes, chez Nievo la bonhommie et le rire l'emportent sur la mélancolie, exactement comme dans les publications périodiques. Il en va de même chez Rajberti, qui qualifie d'humoristique la façon dont il traite son sujet - un voyage à Paris effectué en 1855 en qualité d'ignorant - au moment de prendre congé de son lecteur, alors qu'il dresse la liste des nombreux points restant à traiter, dont il confie le soin à d'autres, en les enjoignant de le faire à sa manière, justement :

À vous autres, qui croyez encore aux cent villes du bel paese : distribuez cette liste aux cent ou deux cents académies littéraires (je recommande en premier l'Arcadia) en les invitant à traiter ces sujets, ou même à n'en choisir que trois ou quatre parmi les plus faciles et géniaux; et à les traiter, bien entendu, de façon plaisante, bizarre, humoristique, tel que je l'ai fait moi-même. À cette fin vous pourriez leur envoyer cet exemplaire du premier tome, que vous êtes en train de lire : ainsi j'aurais moi aussi l'insigne honneur qu'au moins trois cents personnes de plus, présidents, secrétaires et balayeurs, apprendraient mon nom ${ }^{52}$.

Le motif avoué - en tout cas affiché - de l'écriture du Viaggio est la quête de célébrité et de recettes financières («mon faible est justement là : la monomanie de devenir célèbre à tout prix $^{53} »$ ), objectifs qu'un livre sérieux, écrit à force de longues heures de travail, ne saurait atteindre, alors qu'il suffit de décocher quelques flèches :

Avec un tout petit livre de rien du tout, cancanier, impertinent, mordant, on suscite plus de bruit et de scandale qu'avec les gros livres écrits à la lueur d'une lampe à huile phtisique ${ }^{54}$.

La satire reste donc au cœur de l'écriture humoristique, mais le ton facétieux est, chez Rajberti aussi, une condition sine qua non.

Or, la tradition critique ultérieure n'a eu de cesse de vanter la suprématie de la composante sérieuse de l'humour, notamment de l'humour italien. Il en va ainsi des tout premiers jugements sur la question émis par Dossi, De Sanctis et Pirandello ${ }^{55}$; il en va de même des critiques littéraires, qui s'efforcent de distinguer l'humour à dominante joyeuse, mais de mauvais aloi, c'est-à-dire celui de la presse satirique, de l'humour à dominante sérieuse, de bon aloi - celui des écrivains. Ainsi Anna Scannapieco inscrit-elle par exemple les hebdomadaires humoristiques qui voient le jour au milieu du XIXe siècle dans la Trivialliteratur - c'est-à-dire la littérature de gare ${ }^{56}$. Il n'est pas jusqu'aux journalistes eux-mêmes, y compris ceux qui se servent des techniques humoristiques, qui ne critiquent le genre. C'est surtout le cas de Cletto Arrighi dans la Cronaca grigia, dont le programme annonce vouloir se démarquer autant du « style ambigu de certains journaux humoristiques » que « du froid jargon de certains journaux sérieux ${ }^{57}$ ». 
Ayant lu dans les annonces que Sonzogno s'apprête à publier le Spirito Folletto, journal humoristico-satanique, etc., avec caricatures, l'Unità Italiana s'est mise à redoubler de zèle et d'humour pour ne pas se laisser devancer. Et hier, entre autres choses facétieuses, elle en a trouvé une assez spirituelle : elle est sortie en deuil pour l'anniversaire de la cession de Nice et de la Savoie.

Ah! Ces pauvres journaux humoristiques ne pourront jamais faire fortune tant que durera la très puissante concurrence de l'Unità Italiana ${ }^{58}$.

Quatre ans auparavant, nous savons qu'il avait déjà écrit dans L'uomo di pietra :

les Anciens connaissaient tout, sauf peut-être le cosmétique hongrois pour fixer les moustaches, et cet absurde style d'écriture qu'on appelle humoristique, dont on me dit qu'il plaît beaucoup au public cultivé, et que j'appellerais personnellement sot et grotesque si ne j'avais pas de bonnes raisons de dissimuler mes sentiments en la matière ${ }^{59}$.

Un style "absurde », "sot et grotesque », estime Righetti (le vrai nom du journaliste). Pourtant, lui-même fait usage d'humour, de ce même humour qui était déjà à l'œuvre dans les "pauvres journaux humoristiques" auxquels -étrangement - il avait assidument collaboré ${ }^{60}$. Telle est sans doute la raison qui le poussait à « dissimuler » ses sentiments. On ne trouve pas de tels remords chez Giovanni Rajberti, loin s'en faut. Le médecin-poète, comme il se plaisait à se présenter, revendique au contraire son amitié avec les journalistes des publications humoristiques et va même jusqu'à déclarer leur vis comica supérieure à la sienne :

À vous autres, mes chers: Pasquino, Pungolo, Uomo di Pietra! Si j'ai dit que j'étais le seul capable de faire rire, je voulais parler des livres: mais dans les journaux hebdomadaires, vous êtes inégalables : et vous ne pouvez pas imaginer avec quelle tendresse j'embrasse en pensée Fra Fusina, Dottor Bugia, Pif, Brrrr, Vattelapesca ${ }^{61}$ !

L'origine infamante de l'humour, produit d'importation, né dans un contexte éditorial de bas étage, caractérisé par le comique, la légèreté, l'hétérogénéité, explique aisément pourquoi les essayistes, historiens de la littérature, critiques, et les auteurs eux-mêmes, dans leurs écrits théoriques, passèrent sous silence les premières manifestations avouées d'humour dans la presse satirique et valorisèrent les aspects graves, littéraires et philosophiques de l'écriture humoristique. Certes, les visées satiriques d'ordre politique sont centrales, tout comme la polémique contre la dépravation des mœurs, le déclin des idéaux ${ }^{62} . \mathrm{Si}$, toutefois, un hebdomadaire comme $\mathrm{La}$ lente les traite surtout avec sérieux - comme lorsqu'il déplore la corruption du « siècle des lumières, du siècle du progrès, du siècle de la civilisation ${ }^{63} »-$, la plupart des journaux privilégient un ton résolument facétieux. N'en déplaise aux tenants d'une noble généalogie, l'humour déclaré naît donc comme un instrument polémique mais aussi, et surtout, de divertissement, intimement lié à une jouissance immédiate ou limitée à une très brève échéance (la conversation de salon, la publication hebdomadaire), relégué aux marges de l'activité sociale (la maison villégiature, les sujets anodins) dans un registre bas et superficiel (la presse illustrée). Il n'en demeure pas moins que c'est dans un tel contexte que germent les ferments d'un certain renouveau culturel ${ }^{64}$. 


\section{NOTES}

1. Effetto Sterne. La narrazione umoristica in Italia da Foscolo a Pirandello, Pisa, Nistri-Lischi, 1990.

2. Roberta Colombi, Ottocento stravagante. Umorismo, satira e parodia tra Risorgimento e Italia unita, Roma, Aracne, 2011.

3. Anna Scannapieco, «Lemmi e "dilemmi" dell'umorismo. Per una morfologia (e storia) della letteratura umoristica in Italia ", Rivista di letteratura italiana, XX, 2, 2002.

4. Cf. par exemple Daniela Marchesi, «Leopardi e l'umorismo », in Il lettore di provincia, n. 130, 2008 ; Luciano Parisi, « L'umorismo di Manzoni », in Italian studies, LVII, 2002.

5. Précisons toutefois que l'usage moderne du lexème renoue avec l'acception inaugurée par les académies des humoristes qui fleurirent au XVIIe siècle. Michele Maylender recense notamment celles de Bondino, de Milan et, bien sûr, de Rome (cf. Michele Maylender, Storia delle accademie d'Italia, vol. V, Bologna, Cappelli, s.d.).

6. Cependant, d'autres journaux que tout porte à inclure dans le genre humoristique ne comportent pas le terme dans leur sous-titre. C'est par exemple le cas de Il fischietto: bizzarrie d'attualità. Rivista illustrata con disegni originali de Turin, dont le premier numéro parut en 1848 . D'après Anna Scannapieco, on dénombre 14 publications humoristiques entre 1848 et 1849, puis les quantités ne cessent d'augmenter jusqu'au début du XXe siècle : 17 titres de 1850 à 1859, 49 de 1860 à 1869 , etc.

7. À partir de 1849 apparaissent également Il gobbo di Rialto. Giornale critico politico umoristico urbano (Venise), Misteri di Roma: giornaletto storico-politico-umoristico-popolare dedicato ai circoli dell'Italia centrale (Rome), Asmodeo il diavolo zoppo : giornale politico-umoristico a benefizio di Venezia (Venise), L'Inferno: giornale della notte: politico-diabolico-bizzarro-letterario-umoristico-fantasticoridicolo-critico-satirico-pittoresco-teatrale: illustrato da mille e più disegni all'anno (Livourne), La zanzara : giornale umoristico con vignette (Florence), Il costituzionale : giornale umoristico (Trieste).

8. Comme substantif, il ne renvoie qu'à la personne : dans les années 1850 on trouve le terme umorista sous la plume de Rajberti et dans L'uomo di pietra. Il faut attendre 1875 pour que le Vocabolario italiano della lingua parlata de Fanfani et Rigutini définisse le terme umorista dans le sens exclusif d'une personne "che ha umore faceto, e scrive cose facete" [qui a l'humeur facétieuse et écrit des choses facétieuses]; le terme umoristico, entendu comme synonyme de faceto [facétieux], fait également son apparition comme attribut de journaux et écrits.

9. Ainsi reçut-elle le tract incitant à la grève des cigares le $1^{\mathrm{er}}$ janvier 1848 - l'événement qui donna lieu ensuite aux Cinque Giornate.

10. "gli antichi conoscevano tutto, tranne forse il cosmetico ungherese pour fixer les moustaches, e questo assurdo genere di scrivere chiamato umoristico, che mi si dice piaccia molto al colto pubblico, e che io chiamerei stolido e grottesco se non avessi delle ragioni per dissimulare i miei sentimenti in proposito " (Cletto Arrighi, «La Marsina ovverosia Coda di rondine. Baggianata cronologica, filosofica, etimologica e morale dedicata ai ballo-maniaci ", in L'uomo di pietra, n. 10, 17 gennaio 1857, p. 78-79). Toutes les traductions de l'italien au français sont de notre fait, exception faite des passages tirés du roman de Nievo.

11. «En anglais, le sens du mot a évolué pour désigner dans le courant du XVIIIe siècle la faculté de présenter la réalité de manière à en montrer les aspects plaisants, insolites ou parfois absurdes, avec une attitude empreinte de détachement» («Humour» [ad vocem], Le Robert. Dictionnaire historique de la langue française, sous la direction d'Alain Rey, Paris, Dictionnaires le Robert, $2000^{3}$, p. 1053). D'autres sources indiquent que la naissance du terme anglais humour date du XVIIe siècle : «Au XVIIe siècle, l'anglais emprunta le mot français [humeur], sous la forme 
humour, pour désigner un tempérament enclin à la plaisanterie, puis la plaisanterie elle-même » («Humour»[ad vocem], René GARRUS, Étymologies du français. Curiosités étymologiques, Paris, Éditions Belon, 1996, p. 192). C'est en effet au XVIIe que sont attestés les premiers usages du terme dans le sens d'une qualité suscitant le rire ou l'amusement: "That quality of action, speech, or writing, which excites amusement ; oddity, jocularity, facetiousness, comicality, fun » («Humour» [ad vocem], The Oxford English Dictionary, prepared by J. A. Simpson and E. S. C. Weiner, vol. VII, Oxford, Oxford University Press, 1991, p. 486).

12. «Le dernier emploi, qui a supplanté les autres, est attesté en 1793 comme adjectif; humoriste est alors une francisation de l'anglais humorist ou humourist [...], emprunté lui-même au français humoriste (dans son premier sens) ou à l'italien umorista et qui a pris la valeur de "personne facétieuse, enjouée" en 1599 » ("Humoriste » [ad vocem], Le Robert. Dictionnaire historique de la langue française, sous la direction d'Alain Rey, Paris, Dictionnaires le Robert, 2000³ , p. 1053).

13. «La cultura italiana, compresa quella milanese, è pressoché estranea alle nuove elaborazioni teoriche » [La culture italienne, y compris la culture milanaise, est presque étrangère aux nouvelles élaborations théoriques] (Alberto Cadioli, Romanticismo italiano, Milano, Editrice Bibliografica, 1995). En particulier, dans le contexte européen, le développement du journalisme $\mathrm{y}$ est très tardif. Dans les premières décennies du siècle, le Quaterly Review comptait environ 10.000 abonnées, tandis qu'aucun journal de la péninsule ne dépassait les 400-500 abonnés. Déplorer la «torpeur » qui sévit dans la culture péninsulaire est d'ailleurs un véritable leitmotiv : Ludovico Di Breme s'exclame «Inerti siam noi »[Nous sommes inertes] et Silvio Pellico renchérit : «Il maggior de' pericoli che sovrasta a questo paese si è il torpore, l'abitudine di non pensare » [Le plus grand péril qui pèse sur ce pays, c'est la torpeur, l'habitude de ne pas penser] (lettre à Foscolo du 17 octobre 1818).

14. Cf. Marino Berengo, Intellettuali e librai nella Milano della Restaurazione, Torino, Einaudi, 1980.

15. «TRUTH was the Founder of the Family, and the Father of GOOD SENSE. GOOD SENSE was the Father of WIT, who married a Lady of a Collateral Line called MIRTH, by whom he had Issue HUMOUR. HUMOUR therefore being the youngest of this Illustrious Family, and descended from Parents of such different Dispositions, is very various and unequal in his Temper; sometimes you see him putting on grave Looks and a solemn Habit, sometimes airy in his Behaviour and fantastic in his Dress : Insomuch that at different times he appears as serious as a Judge, and as jocular as a Merry-Andrew. But as he has a great deal of the Mother in his Constitution, whatever Mood he is in, he never fails to make his Company laugh » (Joseph Addison, The Spectator, n. 35, 10 April 1711, http://www.gutenberg.org/files/12030/12030-h/12030-h/SV1/ Spectator1.html\#section35). Les traductions de l'anglais sont de notre fait. Dans les lignes suivantes, Addison s'attache à différencier le vrai humour du faux humour, lequel est au vrai humour, écrit-il, ce que le singe est à l'homme.

16. «il programma della Lente si riepiloga in due parole : Caricature e Articoli » [le programme de la Loupe tient en deux mots : Caricatures et Articles] (Collodi, "Coda al programma della Lente », in La lente, n. 1, Gennaio 1856, p. 2). Quelques rares journaux humoristiques ne comportent cependant pas d'illustration: La Marmitta de Bologne, par exemple, sous-titré «Giornale umoristico settimanale ", qui était le supplément du Patriota Cattolico à partir de 1865.

17. «- Sei uscito ben per tempo questa mattina, che ho trovato alle nove ore il tuo uscio inchiodato. / - Hai suonato il campanello? / - Per bacco! L'ho strimpellato per mezz'ora. / - Ecco il male. Ti ho preso per un creditore » (« Braciere », in L'uomo di pietra, n. 9, 10 gennaio 1857, p. 71).

18. « È impossibile metter piede in un'isola senza andarvi per acqua. / Impossibile persuadere il Vice-sindaco Bertini a non fare spazzare le vie nelle ore di maggior concorso. / [...] Impossibile fumare uno [sic] sigaro delle R. fabbriche senza avvelenarsi. / Impossibile trovare un francese che non dica chez-nous ad ogni cinque minuti » («Cose impossibili », in Pasquino, n. 19, gennaio 1856, p. 148). L'article est signé ${ }^{* * *}$. 
19. Ainsi Nievo journaliste écrit-il de lui-même, en jouant manifestement sur l'effet d'accumulation: «Sono un letterato, ricco, spiantato, ameno, ipocondriaco, apata, fanatico, caritatevole, egoista, felice disperato come porta l'umore » [Je suis un homme de lettres, riche, déraciné, amène, hypocondriaque, apathique, fanatique, charitable, égoïste, heureux ou désespéré selon l'humeur] (cit. in Roberta Colombi, «L'umorismo che fa 'ridere e piangere e pensare'. Il caso Nievo : narratore e giornalista umorista ", in Scaffale aperto, n. 1, 2010, p. 101). L'activité journalistique de l'écrivain se situe entre 1857 et 1860.

20. Alors Nievo pourra écrire, anticipant la conception que Carlo Dossi aura de l'humour, que la vie n'est « né tutta allegra né tutta tetra, né tutta correvole né tutta irta, né tutta rosea né tutta nera. L'è appunto essenzialmente umoristica per la mescolanza che avviene in essa di tutti questi elementi così disparati » [ni entièrement joyeuse ni entièrement triste, ni entièrement plane ni entièrement rocailleuse, ni entièrement rose ni entièrement noire. En fait, elle est essentiellement humoristique du fait du mélange de tous ces éléments si disparates] (cit. in Ibid., p. 104).

21. À la fin du troisième livre, au chapitre XXXVI du chef-d'œuvre de Sterne, le narrateur affirme que la " marbled page » est " motly emblem of [his] work " (Laurence Sterne, The life and opinions of Tristram Shandy, vol. 1-2, in The complete works and life of Laruence Sterne, vol. 1, New York, AMS Press, 1970, p. 119). Motly, aujourd'hui devenu motley, signifie bigarré, bariolé, hétéroclite, mais en tant que substantif, il désignait autrefois « clothes worn by a jester », c'est-à-dire l'habit du bouffon ("Motley" [ad vocem], Le Robert \& Collins, Paris, Dictionnaires Le Robert, 1987, et Oxford advanced learner's dictionary, Oxford, Oxford University Press, 1989). Cf. Carlo Vita, « Sterne e un ready-made del Settecento. Un percorso di lettura », in Il lettore di provincia, n. 126-127, 2006, p. 40. 22. «Il biennio rivoluzionario 1848-49 si presenta come una particolare contingenza storica durante la quale la ritrovata libertà di stampa e la presenza di opzioni politiche diverse sulla questione nazionale determinarono un orientamento decisamente politico-militante delle numerosissime testate che nacquero in tutti gli Stati italiani. [...] il ritorno della censura sulla stampa costrinse i periodici a ritornare al modello del giornale letterario e di costume » (Ugo M. Olivieri, "Le riviste ", in Manuale di letteratura italiana, storia per generi e problemi, a cura di F. Brioschi e C. Di Girolamo, Torino, Bollati Boringhieri, 1997, p. 131).

23. "Il venir fuori con una Lente nel bel mezzo del Secolo dei Lumi, in cui deve presumersi che tutti gli uomini ci veggano chiaro, e che non sia più possibile il caso, che spesso avveniva alla buon'anima dei nostri Vecchi, di prender lucciole per lanterne, potrebbe per avventura stimarsi impresa inutile od irrisoria » (La lente, n. 1, 1 gennaio 1856, p. 1). L'article est signé Don Bartolo.

24. " un Giornale-Lente apporterà un gran benefizio alla cieca umanità » (Ibid., p. 2).

25. «cercare la verità per la via del ridicolo", "Scuoprire nuovità e pubblicarle ridendo » («Risus omnia vincit », in La lente, n. 3, 15 gennaio 1856, p. 2). L'article est signé Canicola.

26. « scandagliare le piaghe della società » (« Mastro Forese », in Ibid., p. 2).

27. « a cosa giovano i progressi delle civiltà, quando un popolo non possa vestirsi, almeno una volta l'anno, da Arlecchino o da Pulcinella?» [que valent les progrès de la civilisation si un peuple ne peut pas s'habiller, au moins une fois par an, en Arlequin et Polichinelle ?] (« Coda al programma della Lente », in Ibid., p. 1). L'article est signé Collodi. C'est un véritable leitmotiv du journal florentin. De manière moins détournée, un article du mois de mars déplore encore la corruption des mœurs présentes. Tandis qu'autrefois, une poignée de main suffisait à engager deux parties contractantes, «Oggigiorno però, mercè le cognizioni e l'incivilimento, ci vuol altro che stretta di mano! Talvolta non basta neanche un contratto fatto per mano di pubblico notaro, e alla presenza di cento testimoni ! Eh! gli uomini oggidì nascono ad occhi aperti !» [De nos jours, au contraire, grâce aux connaissances et à l'évolution des mœurs, il faut bien autre chose qu'une poignée de main! Parfois, un contrat rédigé par un notaire assermenté et en présence de cent témoins ne suffit même pas! Eh! De nos jours les hommes naissent les yeux grand ouverts !] (« Il mondo peggiora !... », in La lente, n. 11, 11 marzo 1856, p. 1). L'article est signé Don Bartolo. 
28. « Leggiamo. "Carissimo Padre. Finalmente godo un poco di pace. Il frastuono del carnevale, il rumore delle carrozze, lo schiamazzo delle maschere mi concederanno appena le ore della notte per dedicarmi allo studio." / Avvertite che egli ha urlato in maschera tutti i giorni e più di tutti. Inoltre ha ballato tutte le notti fino a giorno. [...] / "I ferri che acquistai cogli ultimi vostri denari..." / Comprò gli sproni per andare a cavallo. / "Sono eccellenti, ma mi ci vorrà della spesa per farli arrotare, giacchè in pochi giorni ho tagliato una dozzina tra gambe e cosce..." / Gambe e cosce di pollo » («Scene quaresimali. Strascichi carnevaleschi », in La lente, n. 7, 12 febbraio 1857, p. 2). L'article est signé Maestro Forese.

29. «Havvi due specie d'inventori. - L'Inventore che nasce inventore, come si nasce poeta od artista, - e l'inventore che diviene inventore, in seguito ad esperienze della vita, dopo aver provato... di tutto. / Il primo è spesso un martire, quando non è un matto. È un originale, che vive d'idee fisse, e di pane e d'acqua. O inventa qualche gran cosa, come Guttemberg [sic] e Watter, o muore all'ospedale senza aver inventato nulla ; cioè - notate - muore all'ospedale in ambedue i casi [...]. / Questo non è l'Inventore mio prediletto, nè il più frequente ai dì nostri. [...] Oggi la prima invenzione cui pon mente sul serio un inventore, è quella di far in modo, - sia il genio o la mariuoleria che gli dieno la spinta, - ch'ei non debba morire all'ospitale. Anzi, che dico ?... Le cose si sono rivoltate al punto, che quasi sempre l'Inventore, tanto più il mariuolo senza genio, muore non già all'ospitale, ma lasciando a' suoi eredi centomila lire per l'erezione d'un ospitale..., che - fra parentesi - viene innalzato coi mattoni degli azionisti i quali, colpa l'Inventore, muoiono spesso all'ospitale in luogo suo » (« Nuovi tipi sociali. L'inventore», in Pasquino, n. 1, 27 gennaio 1856, p. 6). L'article est signé FSCHT.

30. «Vi è un genere di poesia del più alto interesse, che mai non invecchia, e che tra noi modernamente trovò pochi culturi [sic] - la Satira. - Schivando la troppa acrimonia e la licenza del Menzini, astenendosi dalla personalità e dalla trivialità di Salvator Rosa, senza pretendere all'altezza inimitabile e pericolosa del Giusti, la satira si occupi solamente dei cattivi costumi, e li dipinga al vivo, talchè ne nasca ora l'odio, ora il disprezzo, ora il ridicolo " [Il est un genre de poésie du plus haut intérêt, qui ne vieillit jamais, et qui trouva chez nous peu d'adeptes - la Satire -. Que la satire évite l'acrimonie excessive et la licence de Menzini, la personnalité et la trivialité de Salvator Rosa et, sans prétendre à la grandeur inimitable et dangereuse de Giusti, qu'elle s'occupe seulement des mauvaises mœurs, et les dépeigne sur le vif, pour en susciter la haine, le mépris ou le ridicule selon le cas] («Di due specie di Poeti moderni che non sono Poeti », in La lente, n. 4, 22 gennaio 1856, p. 2). Carlo Tenca aussi tenait en grande estime le poète satirique. Cf. Carlo Tenca, « Giuseppe Giusti », in Giornalismo e letteratura nell'Ottocento, a cura di G. Scalia, Rocca San Casciano, Cappelli, 1959, p. 146-155.

31. «Il caoutchouc! - sostanza maravigliosa, sorprendente, filantropica! Sostanza destinata a supplire, col tempo, a tutti i bisogni della vita. [...] / Ma fra tutti i miracoli che l'uomo ha fatto fare fin qui a questo prodotto vegetale, il più grande, il più utile, si è - senz'alcun dubbio - quello della sua applicazione alla letteratura e all'arte libraria. / Ed anche di questo miracolo il mondo civile va debitore alla Francia, alla generosa Francia, alla grande nazione civilizzatrice. [...] / La dinastia degli Alessandri Dumas fu la prima ad applicare il caoutchouc al romanzo. / Alessandro I vi tira giù un romanzo di trecento pagine in ventiquatt'ore [sic]. [...] / Aprite un romanzo qualunque fra i centomila scritti da lui e potrete convincervene. / Esempio : / Marcellina! / Alberto ! / Ah ! / Oh ! / Sei proprio tu ? / Sì. / Tu ? / Davvero. / Gran Dio ! / E qual maraviglia ? / Io ti credeva morta. / Morta? / Morta! / Ah Alberto ! / Oh Marcellina ! / [...] / Alla pagina decimosettima Alberto si persuade finalmente che Marcellina non è morta e finisce il capitolo. / Potenza del caoutchouc!» («Caoutchouc!», in Pasquino, n. 22, 22 giugno 1856, p. 173-174). L'article est signé BRRRR.... ! Un autre article du Pasquino s'en prend à Cesare Cantù : « Vous savez sans aucun doute que Monsieur Cesare Cantù - dès la première édition de sa première œuvre était sujet à de fréquentes douleurs, à de profonds abattements, toujours suivis d'amères désillusions ! / À le voir, on ne croyait pas, parce qu'il se montrait fringant, en bonne santé, et d'humeur 
joyeuse : mais les apparences sont trompeuses; et sous cette apparence, et à chaque nouvelle préface de chaque nouvelle édition de ses œuvres, les douleurs, les abattements et les désillusions qui suivent ne manquent jamais de l'assaillir. [...] / Moi, pour parler franchement, j'ai bien l'impression que ces douleurs de Monsieur Cantù résident dans les préfaces. / Dans ce cas - si j'étais médecin - je conseillerais à Monsieur Cantù de ne plus écrire de préfaces » [Sapete senza dubbio come il sig. Cesare Cantù - fin dalla prima edizione della prima sua opera - andasse soggetto a frequenti dolori, a scoraggiamenti profondi, seguiti sempre da amari disinganni! / A vederlo non pareva, perchè si mostrava vegeto, in buona salute, e in lieto umore : ma l'apparenza inganna ; e sotto a quella superficie, ed in ogni nuova prefazione di ogni nuova edizione delle sue opere, i dolori, gli sconforti, e il seguito dei disinganni non mancano mai d'assalirlo. [...] / Io, a dirla schietta, ho gran sospetto che questi dolori del sig. Cantù abbian sede nelle prefazioni. / In questo caso - se fossi medico - consiglierei al sig. Cantù di non scrivere più prefazioni] («I dolori e gli sfoghi del signor C. Cantù », in Pasquino, n. 23, 29 giugno 1856, p. 178). L'article est signé BTZ.

32. Cletto Arrighi, «Una lezione su Dante e la Divina commedia per M. ${ }^{\mathrm{r}}$ Bess-Thiòn Prof. alla Facoltà delle Lettere di Parigi ", in Cronaca grigia, n. 9, 10 gennaio 1857, p. 70-71. Les spécificités typographiques et orthographiques du texte original - en français - ont été scrupuleusement respectées.

33. Il s'agit parfois de sujets socio-politiques. Un article de La lente est consacré aux enfants abandonnés. Il déplore que nombre de ces orphelins soient pris en charge par des familles dans le seul but de toucher la subvention annuelle que l'hôpital public alloue aux parents adoptifs, sans se soucier des conditions d'hygiène et de moralité de la famille d'accueil. L'attaque est cependant assortie d'une proposition constructive, où le journaliste exprime le souhait que le gouvernement prenne en charge ces enfants en leur apprenant un métier artisanal et en envoyant les garçons composer les garnisons, au grand bénéfice des familles qui souffrent de voir leurs fils quitter le domicile pour servir le drapeau. Cf. « I gettatelli veduti colla lente », in La lente , n. 2, 8 gennaio 1856. L'article est signé F. Da Verghereto.

34. «Lippa Inviato straordinario e Ministro plenipotenziario di S. M. il Re della Cappadocia presso il Senatus Popolusque romanus, esce dal suo palazzo, con passo maestoso e il sigaro in bocca, per recarsi dal Ministro degli affari esteri, al quale doveva comunicare una nota energica del suo Governo, risguardante [sic] una specie di questione d'Oriente di quell'epoca » (« Il nostro programma ", in Pasquino, n. 1, 27 gennaio 1856, p. 1).

35. «Lippa intravvide l'utilità di una risata a tempo; e via via fantasticando sull'efficacia e sull'importanza della missione filosofico-umanitaria del riso, giunse fino all'idea di pubblicare per le stampe un'Effemeride comica con caricature [...].Così ebbe vita il primo giornale umoristico che ricordi la storia » (Ibid., p. 2).

36. «sempre di questo brando a due tagli armato, le cose novelle non tanto, che le già note rifrustar volli » («I gettatelli veduti colla lente », in La lente, n. 2, 8 gennaio 1856, p. 1). L'article est signé F. Da Verghereto.

37. Cette définition est fournie à l'occasion d'une critique élogieuse - une fois n'est pas coutume - de l'adaptation théâtrale du poème satirique de Giuseppe Parini par Paolo Ferrari : "Questa volta l'Uomo di Pietra scrive col cuore e non colla testa... dimentica il suo umorismo... ambrosiano... / A che infatti l'umorismo ? / L'umorismo è una battaglia combattuta a parole, ad arguzie sottili... [...] / L'umorismo è una forte e generosa protesta incontro gli uomini e le cose e le opere male; è rimprovero de' fatali pregiudizii ; è argueta correzione delle false idee; è desiderio del meglio in tutti i rapporti umani fortemente espresso coll'apologo, il simbolo, l'immagine, il geroglifico, il sottinteso ; è condanna pronunciata dall'ideale sconosciuto incontro la turpezza onorata, seguitata ; è fiero cozzo impresso via via alla congerie di tutto che è vieto ed assurdo e vile; - è una guerra di parole e di cose, d'idee e di fatti..." ("La Satira e Parini. Commedia dell'Avv. Paolo Ferrari », in L'uomo di pietra, n. 11, 13 marzo 1858, p. 188). L'article est signé G. D-C. Le drammaturge Paolo Ferrari (1822-1889) conquit le public avec Goldoni 
e le sue sedici commedie, mis en scène en 1853. Il obtint également un grand succès avec La satira e il Parini, représenté pour la première fois en 1856.

38. Il ajoute: "C'est avec ces intentions, entre autres, que naquirent justement chez nous l'Uomo di Pietra, le Pungolo et, sous une forme plus sérieuse que les deux autres, le Crepuscolo. / Ce dernier, fondé en 1849 ? par Carlo Tenca, nom déjà connu dans les lettres pour des ouvrages de qualité, occupera dans l'histoire littéraire de notre temps la même place que celle qu'occupent le Conciliatore et le Caffé dans leurs époques respectives ». "Quella strategia, che, come la militare, ha le sue marcie e contromarcie, le sue imboscate e strattagemmi [sic] e sorprese e finti attacchi, fu chiamata con vocabolo quasi inglese umorismo, il quale quanto più è vivace e leggero nella forma, tanto più è serio nel fondo. Strumento codesto che si dovrà gettare quando la libera frase potrà mettersi esplicita, coraggiosa, intera, in luogo di quella che adesso una stolida censura non ci permette quasi neppure di pensare. In generale si crede che l'umorismo sia quel non so che dell'arte per mezzo del quale si fanno ridere i lettori. Sì; anche il riso a suo tempo è una bella cosa, e felici gli scrittori che a suo tempo sanno suscitarlo. Però questo sarà tutt'al più una maniera speciale dell'umorismo; ma non comprende intero il concetto di esso. [...] / Anche la forma umoristica servì a rendere in questi ultimi anni la letteratura milanese. Il pericolo continuo di esser colpiti in cui stanno i giornali militanti, quell'ansia nei lettori di scoprire la significazione nascosta nelle frasi che sono già passate sotto gli occhi degli stolidi commissarii di censura, e tutto in fine quel lavoro di interpretazione che rende per così dire il pubblico che legge, collaboratore e complice dei giornalisti... tutto contribuisce a vivificare e a tener desto lo spirito letterario nella mia schiava città. / Con tali ed altre intenzioni nacque appunto fra noi l' Uomo di Pietra, il Pungolo, e con veste più seria d'entrambi il Crepuscolo. / Quest'ultimo giornale fondato nel 1849 ? da Carlo Tenca, nome già conosciuto nelle lettere per lavori di merito, prenderà nella storia letteraria dell'epoca nostra quello stesso posto che in altri tempi ebbero il Conciliatore e il Caffe » (Cletto Arrighi, Cinque mesi. Memorie d'un ex repubblicano, in La Cronaca Grigia, n. 5, 29 dicembre 1860, p. 24-27).

39. « Io non sono totalmente del parere del mio ex-repubblicano. L'umorismo può giovare anche a stampa libera, nel senso che può aprire la via a certe idee che i giornali serj e prudenti non avrebbero il coraggio di emettere, e tanto meno di propugnare. Io scommetto che la Perseveranza non oserebbe oggi parlare con tanta libertà delle cose più sacre nel vecchio sistema - se già da molto tempo il Fischietto, l'Uomo di Pietra ed il Pungalo [sic] (umoristico) non le avessero aperta la via, distruggendo col ridicolo il prestigio che certi nomi esercitavano ancora sulla massa delle popolazioni. Col loro umorismo i giornali di caricature preparano per così dire gli animi ad un linguaggio ufficiale, più passionato e più efficace, e si potrebbero per ciò chiamarli avantcoureurs dell'opinione pubblica » (« Nota », in Ibid., p. 24-25). La note est signée C. A.

40. «la qualifica di umoristico viene di volta in volta ricondotta alla coscienza della crisi, all'espressione della libertà e della democrazia, alla cultura dell'antinomia e del paradosso » [le qualificatif d'humoristique est progressivement utilisé en rapport avec la conscience de la crise, l'expression de la liberté et de la démocratie, la culture de l'antinomie et du paradoxe] (Anna Scannapieco, cit., p. 98).

41. "Les événements de 1848 marquèrent en effet une césure dans l'histoire du modèle sternien, grâce justement à l'intervention magistrale de Carlo Collodi: le journalisme comiquehumoristique, tel que l'écrivain toscan l'interpréta et le recréa, prit la voie de la satire, du comique, du rire, du paradoxe, d'une créativité imaginative débordante " [Gli eventi del 1848 segnarono infatti une cesura nella storia del modello sterniano e proprio grazie alla magistrale rielaborazione di Carlo Collodi : il giornalismo comico-umoristico, così come lo interpretò e ricreò lo scrittore toscano prese la via della satira, del comico, del riso, del paradosso, di una dirompente invenzione fantastica] (Daniela Marcheschi, « Leopardi e l'umorismo », in Il lettore di provincia, n. 130, 2008, p. 157).

42. «Scene quaresimali. Strascichi carnevaleschi », in La lente, n. 7, 12 febbraio 1857, p. 2. 
43. «La signora madre è stata ier sera al teatro, dove si rappresentò un dramma francese. Tornata a casa, nel silenzio della notte, nel buio della camera, quelle scene le ripassarono dinanzi agli occhi della mente. (Apro una parentesi. Posso io in buona coscenza [sic] dar gli occhi alla mente ?... Perchè no !... Petrarca, Dio gli perdoni i Petrarchisti, le ha dato ben le ginocchia... Tranquillo, chiudo la parentesi)» («Le punture di spilla. Parole gettate al vento. Fisiologie, Anfibologie, Parodie, Bizzarrie, Annotazioni, Confutazioni, Ammonizioni, ed altre osservazioni. II », in La lente, n. 8, 19 febbraio 1856, p. 3.

44. «E il marito ?... direte voi, leggitori intelligentissimi? Il marito ha fatto un viaggetto in Francia, ed è tornato vestito come l'ultimo figurino» (Ibid., p. 3).

45. «Un bel mattino del... (l'anno è incerto, ciò che v'ha di certo si è ch'era un bel mattino di maggio, nel qual mese tutti i mattini sono belli - quando non piove) » (« Il nostro programma », in Pasquino, n. 1, 27 Gennaio 1856, p. 1).

46. Ils poursuivent : «La naissance du roman coïncide dès le début avec la naissance de l'antiroman parodique » [il momento antiletterario si trasmuta nel suo opposto e complementare : il momento metaletterario [...]. Al romanzo si affianca fin dall'inizio l'antiromanzo parodico] (Franco Brioschi, Costanzo Di Girolamo, "I generi”, in Manuale di letteratura italiana, a cura di F. Brioschi e C. Di Girolamo, III, Dalla metà del Settecento all'Unità d'Italia, Torino, Bollati Boringhieri, 1995).

47. Le vilain petit canard [Den grimme aelling] (1842) est à l'origine un conte de Hans Christian Adersen.

48. Il poursuit : «En Angleterre, au contraire, elles subissent des mélancolies, se rongent, se passionnent, se tuent. Ce sont deux immoralités, ou deux folies ; mais je ne veux me décider pour aucune des deux " [Chi ha cercato in Inghilterra i creatori dell'umorismo non visse mai certamente a Venezia, né mai passò per Portogruaro. Vi avrebbe trovato, frutto di lunghi ozii secolari, di ottimi stomachi e d'ingegni pronti allegri svegliati quell'umorismo meridionale che tanto si distingue dal settentrionale quanto la nebbia notturna del palude dall'orizzonte lucente e vaporoso d'un bel tramonto d'estate. La vita e le cose che sono in essa, disprezzate ugualmente ; ecco la parentela; ma perciò appunto volte tutte alla spensieratezza e alla gioia; ecco la diversità. In Inghilterra invece danno in melanconie, si rodono, si appassionano, si ammazzano. Sono due immoralità, o due pazzie ; ma non voglio decidermi per nessuna delle due] (Ippolito Nievo, Le confessioni di un italiano, a cura di M. Gorra, Milano, Mondadori, 1981, p. 275).

49. «Il nobile patrizio si diportò colla proverbiale affabilità dei Veneziani. Dal Vescovo all'ortolano nessuno fu fraudato del favore d'un suo sorriso; al primo baciò l'anello, al secondo diede uno scappellotto coll'uguale modestia » (Ippolito Nievo, Le confessioni di un italiano, cit., p. 264-265).

50. «molto bene di sé, e qualche piccolo male di coloro che erano partiti »; "la discrezione veneziana »; « s'accontenta di tagliar i panni senza rodere le carni fino all'osso » (Ippolito Nievo, Le confessioni di un italiano, cit., p. 265).

51. «la piccola accademia del Senatore si raccoglieva in un angolo del salone a cianciar di politica, e a motteggiare sulle novelle più scandalose della città. Era una musica la più variata, una vera opera semiseria, piena di motivi ridicoli e sublimi, buffi e serii [...]; e a così gravi ragionamenti non si cessava dall'alternare lo scherzo, e la risata. Ogni appiglio per burlare era buono » (Ippolito Nievo, Le confessioni di un italiano, cit., p. 274-275).

52. [A voi altri, che credete ancora nelle cento città del bel paese : diramate quell'elenco alle cento o duecento accademie letterarie (raccomando per prima l'Arcadia) con invito a trattare quelli argomenti, o anche solo a sceglierne tre o quattro dei più facili e geniali ; e trattarli, bene inteso, in modo scherzevole, balzano, umoristico, a imitazione del mio fare; al quale scopo potreste mandar loro quest'esemplare del tomo primo, che state leggendo : così avrei anch'io l'alto onore che almeno trecento persone di più tra presidenti, secretarii e spazzini imparerebbero il mio 
nome] (Giovanni Rajberti, Viaggio di un ignorante ossia ricetta per gli ipocondriaci, Milano, Rizzoli, 1962, p. 167).

53. [il mio debole sta proprio in ciò : la monomania di rendermi celebre a qualunque costo] (Ibid., p. 24-25).

54. [Con un libercolino smilzo, pettegolo, petulante, pungente, si leva più rumore e scandalo che coi libracci elaborati al lume della tisica lucerna] (Ibid., p. 18).

55. Cf. Roberta Colombi, Ottocento stravagante. Umorismo, satira e parodia tra Risorgimento e Italia unita, Roma, Aracne, 2011, p. 28-32.

56. Les dictionnaires eux-mêmes tendent à éluder la composante facétieuse. De nos jours, le Grande dizionario della lingua italiana Salvatore Battaglia en XXI volumes définit ainsi l'humour : «Capacité à percevoir et présenter la réalité en mettant en relief, par l'exercice d'une distance critique, ses aspects divertissants et insolites, parfois absurdes» [Capacità di percepire e presentare la realtà mettendo in risalto, con un atteggiamento improntato al distacco critico, gli aspetti divertenti e insoliti, talvolta assurdi] («Umorismo» [ad vocem] Salvatore BattagliaA, Grande dizionario della lingua italiana, Torino, UTET, t. XXI, 2002, p. 531).

57. Cletto Arrighi, La Cronaca Grigia, n. 1, 1860, p. 6. Sur Cletto Arrighi et la Cronaca Grigia, cf. Giulio Carnazzi, Da Rovani ai «Perduti». Giornalismo e critica nella Scapigliatura, Milano, Edizioni Universitarie di Lettere Economia Diritto, 2002.

58. L'Unità Italiana avendo letto negli avvisi che Sonzogno si appresta a pubblicare lo Spirito Folletto, giornale umoristico-satanico, ecc., con caricature, s'è messa a raddoppiar di zelo e di umorismo per non lasciarsi soppiantare. E jeri, tra le altre cose da ridere, ne ha pensata una abbastanza spiritosa : è uscita a lutto per l'anniversario della cessione di Nizza e Savoja. / Ah ! Questi poveri giornali umoristici non potranno mai far fortuna finchè vive la potentissima concorrenza dell'Unità Italiana » (Cletto Arrighi, La Cronaca Grigia. Pubblicazione settimanale, n. 27, 1 giugno 1861, p. 22).

59. Cf. supra. Cletto Arrighi, «La Marsina ovverosia Coda di rondine. Baggianata cronologica, filosofica, etimologica e morale dedicata ai ballo-maniaci ", in L'uomo di pietra, n. 10, 17 gennaio 1857, p. 78-79.

60. Ainsi trouve-t-on par exemple une parabole visant à fustiger les gouvernants, sous une allure facétieuse. Cf. Cletto Arrighi, "Una parabola", in La Cronaca Grigia, n. 10, 2 febbraio 1861, p. 12-14. Les différentes nations européennes se présentent à Dieu pour Lui présenter leurs requêtes. L'Italie demande beaucoup de beautés et, pour finir, un bon gouvernement national. Dieu, estimant lui avoir donné suffisamment de bienfaits, rejette cette ultime requête.

61. [A voi altri, miei bravi : Pasquino, Pungolo, Uomo di Pietra! Se dissi ch'io sono il solo capace di far ridere, ho inteso sui libri : ma sui fogli volanti, ebdomadarii, gli inarrivabili siete voi : né potete imaginare con che tenerezza io abbracci nel desiderio il Fra Fusina, il Dottor Bugia, il Pif, il Brrrr, il Vattelapesca !] (Giovanni Rajberti, Viaggio di un ignorante, cit., p. 175).

62. La crise qui alimente l'émergence de l'humour, loin d'être exclusivement politique, est essentiellement culturelle. Les deux aspects sont sans aucun doute mêlés chez Nievo, dont l'esprit humoristique vise la condamnation «de l'évolution capitaliste du monde moderne » [dell'evoluzione capitalistica del mondo moderno], un monde « où tout devient petit et mesquin » [dove tutto diventa piccolo e gretto] (Roberta Colombi, «L'umorismo che fa "ridere e piangere e pensare". Il caso Nievo : narratore e giornalista umorista ", in Scaffale aperto, n. 1, 2010, p. 102).

63. «Oggigiorno però, mercè le cognizioni e l'incivilimento, ci vuol altro che stretta di mano! Talvolta non basta neanche un contratto fatto per mano di pubblico notaro, e alla presenza di cento testimoni ! Eh ! gli uomini oggidì nascono ad occhi aperti ! ( " Il mondo peggiora !... », in La lente, n. 11, 11 marzo 1856, p. 1). L'article est signé Don Bartolo.

64. Certains observateurs l'ont relevé ponctuellement: "è tra i collaboratori dei giornali umoristici che si potranno trovare le personalità forse più aperte al cambiamento e forse più interessate a tradurre il loro spirito critico in nuove forme letterarie " [c'est parmi les 
collaborateurs des journaux humoristiques que l'on pourra trouver les personnalités peut-être les plus ouvertes au changement et les plus intéressées à traduire leur esprit critique en de nouvelles formes littéraires] (Roberta Colombi, Ottocento stravagante, cit., p. 38). Néanmoins ce vaste terrain d'étude attend encore une enquête de fond, qui permettrait également de réévaluer le rôle que jouèrent certaines personnalités, comme Giovanni Rajberti notamment.

\section{RÉSUMÉS}

Qu'entend-on en Italie par humour au moment où le terme est introduit dans l'usage et les dictionnaires, à la moitié du XIXe siècle ? La présente étude porte sur un corpus comprenant trois périodiques autoproclamés humoristiques (La lente de Florence, le Pasquino de Turin et L'uomo di pietra de Milan) et deux romans (les Confessioni di un italiano d'Ippolito Nievo et le Viaggio di un ignorante, ossia ricetta per gli ipocondriaci de Giovanni Rajberti), couvrant les années 1856 à 1860. Il en ressort que, à cette époque - antérieure à l'essor d'un genre littéraire dit humoristique -, l'humour qualifie un genre journalistique satirique, un style d'écriture hybride, un ton facétieux. La prépondérance manifeste du rire impose ainsi une révision de la tradition critique selon laquelle le cœur de l'humour italien est sérieux.

Che cosa si intende per umorismo in Italia quando il vocabolo compare nell'uso e nei vocabolari, alla metà dell'Ottocento? Il presente studio verte su un corpus comprendente tre periodici autorivendicati umoristici (La lente di Firenze, il Pasquino di Torino e L'uomo di pietra di Milano) e due romanzi (le Confessioni di un italiano d'Ippolito Nievo e il Viaggio di un ignorante, ossia ricetta per gli ipocondriaci di Giovanni Rajberti), e va dal 1856 al 1860. Ne deriva che in quel periodo - anteriore all'affermarsi di un genere letterario umoristico - l'umorismo caratterizza un genere giornalistico satirico, uno stile ibrido, un tono faceto. La prevalenza manifesta del riso impone una revisione della tradizione critica secondo cui il nocciolo dell'umorismo italiano è serio.

\section{INDEX}

Index chronologique : XIXe siècle

Mots-clés : humour, satire, presse, journalisme

Index géographique : Italie

\section{AUTEUR}

\section{EDWIGE COMOY FUSARO}

Maître de Conférences Habilitée à Diriger des Recherches à l'Université Nice Sophia Antipolis, Edwige Comoy Fusaro travaille essentiellement sur la littérature, la littérature médicale et l'histoire des idées au XIXe siècle. Elle est l'auteur de deux ouvrages : La nevrosi tra medicina e letteratura. Approccio epistemologico alle malattie nervose nella narrativa italiana (1865-1922) (Firenze, Polistampa, 2007) et Forme e figure dell'alterità. Studi su De Amicis, Capuana e Camillo Boito (Ravenna, Giorgio Pozzi editore, 2009), et s'apprête à publier une étude inédite sur Carlo Dossi. En tant que 
membre du LIRCES, elle a dirigé le séminaire « Humour et Modernité » (2012-2013) et, dans le cadre du séminaire « Littérature et Sciences », la journée d'études « Littérature et Médecine » (2008). 